\title{
Study on the Influence of Physicochemical Indexes on Wine Quality
}

\author{
Simin Wang ${ }^{1, a, ~}{ }^{*}$, Siyuan Jiang ${ }^{2, b}$ \\ 1. School of Physics, Northwest University, Xi'an, China \\ 2. School of Economics and Management, Northwest University, Xi'an, China \\ a, * wangsimin@stumail.nwu.edu.cn, b1021228956@qq.com
}

\begin{abstract}
The quality of a wine is generally determined by hiring a group of qualified wine judges to taste the wine. The quality of wine grapes is directly related to the quality of wine. To explore how the physical and chemical indicators of wine and wine grapes can reflect the quality of wine and grape to a certain extent. This article is based on 2012 Chinese college students' mathematical contest in modeling A problem of data, to score as the dependent variable, wine physical and chemical indicators as independent variables, the method of using principal component analysis of physical and chemical indexes of wine grape dimension reduction, physical and chemical indexes selection of grapes, and use the decision tree regression method to establish score and wine function relation between the physical and chemical indicators. Then, after the comparison of the results of the four clustering algorithms, the hierarchical clustering method based on Agnes algorithm was used to conduct cluster analysis on the wines. Considering the rationality of classification, red and white wines were divided into four grades, and the average value of the estimated scores of each grade was used as the benchmark score for the wines of this grade.
\end{abstract}

Keywords: Principal Component Analysis; AGNES Hierarchical Clustering; Decision Tree Regression.

\section{Problem Description}

The quality of a wine is generally determined by employing a group of qualified wine judges to taste it. Each wine reviewer, after tasting the wine, scores its classification index, and then sums up its total score to determine the quality of the wine. The quality of wine grapes is directly related to the quality of wine, and the physical and chemical indexes of wine and wine grapes can reflect the quality of wine and grape to a certain extent. Attachment 1 shows the evaluation results of some wines of a certain year, and Attachment 2 shows the composition data of these wines and wine grapes of the same year. Please try to build a mathematical model to discuss the following problems:

First, select appropriate physical and chemical indexes for comprehensive evaluation of wine grapes.

Second, analyze the effects of wine grapes and physical and chemical indexes on the quality of wine, and demonstrate whether grapes and wine can be used. The physical and chemical indicators of wine to evaluate the quality of wine.

Third, Wine grapes are graded according to their physical and chemical properties and the quality of the wine.

\section{Problem Analysis}

\subsection{Problem 1 Analysis}

Combined with the data given in the Problem, we considered reducing the dimension of the physical and chemical indexes of wine grapes through the method of principal component analysis, and obtained the comprehensive score of wine grapes based on PAC. 


\subsection{Problem 2 Analysis}

At the same time, we also performed PCA dimension reduction on the physical and chemical indicators of wine to obtain the main explanatory variables. The mean value of the subjective scores of 10 wine tasters was taken as the explained variable. Combining with the physicochemical components of wine grapes in Problem 1, the decision tree regression method was adopted to analyze how the physicochemical indexes of wine grapes and wine affect the quality of wine.

\subsection{Problem 3 Analysis}

It is required to analyze and determine a reasonable evaluation index system, and use the evaluation index system to grade wine grapes.Obviously, this problem requires us to build an evaluation model.Since the quality of wine grapes directly determines the quality of wine, and the quality of wine can be directly reflected and graded through the rating table, so the key problem is how to evaluate the quality of wine grapes.The hierarchical clustering method based on Agnes algorithm carried out cluster analysis according to the comprehensive score of wine grapes and the physical and chemical indexes of wine, and obtained four grades of grapes and benchmark scores of each grade according to the mean of the subjective score of wine produced.

\section{Model Assumption}

First, assume that the various instincts are true and objective reflections of wine grapes and wine;

Second, the quality of wine is only related to the quality of wine grapes, ignoring the influence of temperature, humidity, human interference and other factors during the brewing process;

Third, better wine grapes make better wine;

Finally, second-level indicators without considering physical and chemical properties.

\section{Symbol Description}

Table 1. Symbol Description

\begin{tabular}{|c|c|c|}
\hline Sequence Number & Symbol & Paraphrase \\
\hline 1 & $\lambda_{i}$ & The ith eigenroot \\
\hline 2 & $W_{i}$ & The variance contribution of the ith feature \\
\hline 3 & $T_{i}$ & The ith eigenvector \\
\hline 4 & $M_{i}$ & Comprehensive score for the jth sample \\
\hline 5 & $n$ & Sample size \\
\hline 6 & $X$ & Physical and chemical indexes of wine grape \\
\hline 7 & $Y$ & Physical and chemical indicators of wine \\
\hline 8 & $F$ & Mean subjective score of tasters \\
\hline
\end{tabular}

\section{Model Establishment and Solution}

Problem 1 requires us to establish a model that can classify wine grapes according to their own physical and chemical indexes and the physical and chemical components of the wine after brewing.

To address this problem, we evaluated and graded wine grapes in the following steps.

Firstly, the dimensions of physical and chemical indexes of wine grapes were reduced by principal component analysis method, and the comprehensive score of wine grapes based on PAC was obtained.

Secondly, PCA dimension reduction was also performed on the physical and chemical indicators of wine to obtain the main explanatory variables.

Combined with the variables in the first question, regression analysis was performed on the subjective scores of the wines. 
Finally, based on the hierarchical clustering method, the cluster analysis was carried out according to the physical and chemical indexes of wine grapes and wine, and the grade four grapes and grade benchmark scores were obtained.

\subsection{Data Pre-processing}

After searching for the data, we found that some of the original data were abnormal, and some types of data were missing, so we normalized them here.

\subsubsection{Processing of Missing Data}

For the missing phenomenon in the data, this paper uses the mean substitution method to deal with the missing data.The mean replacement method is to replace the abnormal or missing data by rounding the average value of the remaining data after eliminating the abnormal data of the project, namely:

$$
X_{m}^{*}=\frac{1}{9}\left[\sum_{k=1, k \neq m}^{10} X_{k}\right] \quad(\mathrm{m}=1,2, \cdots, 10)
$$

Where, $X_{m}^{*}$ is the missing value.

Since there is little difference in the scores of the same items from the same sample by different tasters, it is considered feasible to use the mean substitution method to deal with missing data.

Taking the tonal data of 'wine sample 20' as an example to repair, the revised data are shown in the following table:

Table 2. Repair of 20-tone data for red wine samples

\begin{tabular}{|c|c|c|c|c|c|c|c|c|c|c|}
\hline Wine Taster & No.1 & No.2 & No.3 & No.4 & No.5 & No.6 & No.7 & No.8 & No.9 & No. 10 \\
\hline Before repairing & 6 & 6 & 4 & -- & 6 & 6 & 8 & 6 & 6 & 8 \\
\hline After repairing & 6 & 6 & 4 & 6 & 6 & 6 & 8 & 6 & 6 & 8 \\
\hline
\end{tabular}

\subsubsection{Correction of Abnormal Data}

In the original data, some data are obviously too large or too small than the data on both sides, which is obviously unreasonable data.For example, in the first set of white wine tasting score data, perhaps due to manual input errors, the data of taster 7 on the persistence score of sample 3 had a significant mutation relative to the scores of adjacent tasters. This kind of data anomaly may adversely affect the results of data mining.

Table 3. Abnormal persistence data in sample 3 of normal score for white wine

\begin{tabular}{|c|c|c|c|c|c|c|c|c|c|c|}
\hline Wine Taster & No.1 & No.2 & No.3 & No.4 & No.5 & No.6 & No.7 & No.8 & No.9 & No. 10 \\
\hline After repairing & 7 & 5 & 7 & 5 & 6 & 7 & 7 & 5 & 6 & 7 \\
\hline
\end{tabular}

For similar abnormal data, the strategy of "remove first, replace later" is adopted to correct the abnormal data.

\subsection{Dimension Reduction of Physical and Chemical Indexes of Wine Grapes}

For wine grapes, although each indicator is different from each other in the cause of formation, different indicators are often correlated with each other. The reason for this is that there are potential factors that play a dominant role in each indicator of wine grapes.In order to find out these potential factors and their corresponding dominant effects, this paper uses principal component analysis to solve these problems. The steps are as follows:

Step 1: In order to eliminate the dimensionless influence of different variables, the variables need to be standardized first. This part involves the measure of a total of 27, 27 sample objects, The ith index of the jth sample is $F_{i j}$, The normalized values are normalized into $F_{i j}$ as follows: 


$$
\widetilde{F_{i j}}=\frac{F_{i j}-\overline{F_{i j}}}{S_{i}}
$$

Where, $\overline{F_{i j}}$ and $S_{i}$ are the mean and standard deviation of index $i$ respectively.

The purpose of standardization is to eliminate the dimensionless influence of different variables, and the normalization transformation does not change the correlation coefficient of variables.

Step 2: Calculate eigenvalues and eigenvectors. Calculate the eigenvalue $\lambda_{1}>\lambda_{2}>\cdots>\lambda_{27}>0$ of the correlation coefficient matrix $\mathrm{R}$ and its corresponding $T_{1}, T_{2}, \cdots, T_{27}$, where $T_{1}=\left(\mu_{i 1}, \mu_{21}, \cdots, \mu_{27 i}\right)^{T}$. 27 new indicator variables are composed of eigenvectors:

$$
\left\{\begin{array}{c}
Y_{1}=\mu_{11} F_{1}+\mu_{21} F_{2}+\cdots+\mu_{271} F_{27} \\
Y_{2}=\mu_{12} F_{1}+\mu_{22} F_{2}+\cdots+\mu_{272} F_{27} \\
\vdots \\
Y_{27}=\mu_{127} F_{1}+\mu_{227} F_{2}+\cdots+\mu_{2727} F_{27}
\end{array}\right.
$$

Step 3: determine P principal components with sufficient contribution to cumulative variance and conduct statistical analysis.

\subsection{Comprehensive Scoring Model for Wine Grapes}

Principal component analysis (PCA) can summarize most information from the selected index system and conduct comprehensive evaluation according to the information provided by PCA.This method is objective weighting according to the relative importance of indicators, which can avoid the subjective influence of comprehensive evaluation and the dimensional disaster of data. [1]

For the weighted synthesis of principal components, when we use principal components to carry out comprehensive evaluation, we mainly integrate the original information. Therefore, the information provided by the original variables should be fully utilized.The weights of principal components are determined according to their variance contribution rate, because the variance contribution rate reflects the information content of each principal component. Suppose $p$ principal components are obtained, and their characteristic roots are $\lambda_{1}, \lambda_{2}, \cdots, \lambda_{p}$, respectively. After normalization of the characteristic roots, their respective variance contribution weights can be obtained:

$$
W_{i}=\frac{\lambda_{i}}{\sum \lambda_{i}}
$$

Assume that $M_{j}$ is the comprehensive score of the jth sample. Then, based on $Y=T^{\prime} X$, the comprehensive evaluation function is constructed as follows:

$$
\left\{\begin{array}{c}
M_{i}=\sum W_{i} T_{i}^{*} \widetilde{F}_{i} \\
T_{i}^{*}=\frac{T_{i}}{\sqrt{\lambda_{i}}} \\
W_{i}=\frac{\lambda_{i}}{\sum \lambda_{i}}
\end{array}\right.
$$

$M_{j}$ can be used to evaluate the comprehensive score of wine grapes. 


\subsection{Dimension Reduction of Physical and Chemical Indexes of Wine}

The model is similar to the dimension reduction of physical and chemical indexes of wine grape in 5.1.2, so it is omitted.

\subsection{Regression Model for Subjective Rating of Wine}

In order to study how the physicochemical indicators of wine grapes and wines affect the quality of wine, we took the mean value of the subjective scores of 10 wine tasters as the explained variable, and combined with the physicochemical components of wine grapes in Question 1, a regression model was established. [2]

In this paper, we use decision tree regression model and support vector machine regression model respectively, and choose the better model by comparing the prediction errors on the test set.

\subsubsection{Regression of Decision Tree}

We used CART(Classification and Regression Tree) decision Tree algorithm to perform Regression for subjective wine scores. As explanatory variables, the physicochemical indexes of wine grapes obtained through PCA in 5.1.2 and the physicochemical indexes of wine in 5.1.4 were obtained.

The CART decision tree uses Gini index to select partitioning attributes, and the purity of data set $\mathrm{D}$ can be measured by the Gini value:

$$
\operatorname{Gini}(D)=\sum_{k=1}^{|y|} \sum_{k^{\prime} \neq k} p_{k} p_{k}^{\prime}
$$

Intuitively, this model Gini (D) reflects the probability that two samples are randomly selected from data set $\mathrm{D}$ and their category markers are inconsistent. Therefore, the smaller Gini (D) is, the higher the purity of data set $\mathrm{D}$ is.

Represented by the same symbol as the above equation, the Gini coefficient of an attribute is defined as:

$$
\operatorname{Gina} \_ \text {indes }(D, a)=\sum_{v=1}^{v} \frac{\left|D^{v}\right|}{D} \operatorname{Gini}\left(D^{v}\right)
$$

Therefore, in the candidate attribute set A, we choose the attribute that makes the Gini index minimum after partition as the optimal partition attribute, namely:

$$
a_{*}=\arg \min G i n i_{-} \operatorname{index}(D, a)
$$

In this way, we get the regression decision tree model based on CART algorithm.

\subsubsection{Support Vector Machine Regression [3]}

In the Regression of Support Vector machine (SVR), given training sample:

$$
D=\left\{\left(\mathrm{x}_{1}, \mathrm{y}_{1}\right)\left(\mathrm{x}_{2}, \mathrm{x}_{3}\right) \cdots\left(\mathrm{x}_{m}, \mathrm{y}_{m}\right)\right\}, \mathrm{y}_{i} \in R
$$

For traditional regression models, the loss is usually calculated directly based on the difference between the model output $f(x)$ and the real output $y$, and the loss is zero if and only if $f(x)$ and $y$ are exactly the same.SVR is assuming we can tolerate between $\mathrm{f}(\mathrm{x})$ and the y maximum deviation, that is the loss is calculated only if the absolute value of the difference between $f(x)$ and $y$ is greater than $\varepsilon$.

Therefore, the SVR problem can be formalized into:

$$
\min _{w, b} \frac{1}{2}\|w\|^{2}+C \sum_{i=1}^{m} \xi_{\varepsilon}\left(f\left(x_{i}\right)-y_{i}\right)
$$

Where, $\mathrm{C}$ is the regularization constant and $\xi_{\varepsilon}$ is the insensitive loss function of $\varepsilon$ : 
Volume 15 (2021)

$$
\xi_{\varepsilon}(z)=\left\{\begin{array}{c}
0, \text { if }|z|<\varepsilon \\
|z|-\varepsilon, \text { otherwise }
\end{array}\right.
$$

Then, the Lagrange function of the above equation is obtained by introducing Lagrange multiplier:

$$
\begin{aligned}
& L(w, b, \alpha, \hat{\alpha}, \xi, \hat{\xi}, \mu, \hat{\mu})=\frac{1}{2}\|w\|^{2}+C \sum_{i=1}^{m}\left(\xi_{i}+\hat{\xi}_{i}\right)-\sum_{i=1}^{m} \mu_{i} \xi_{i}-\sum_{i=1}^{m} \hat{\mu}_{i} \hat{\xi}_{i}+ \\
& \sum_{i=1}^{m} a_{i}\left(f\left(x_{i}\right)-y_{i}-\varepsilon-\xi_{i}\right)+\sum_{i=1}^{m} \hat{a}_{i}\left(f\left(x_{i}\right)-y_{i}-\varepsilon-\hat{\xi}_{i}\right)
\end{aligned}
$$

The dual problem of SVR is obtained by solving the Lagrange function:

$$
\begin{gathered}
\max _{\alpha, \hat{\alpha}} \sum_{i=1}^{m} y_{i}\left(\hat{\alpha}_{i}-\alpha\right)-\varepsilon\left(\hat{\alpha}_{i}-\alpha\right)-\frac{1}{2} \sum_{i=1}^{m} \sum_{j=1}^{m}\left(\hat{\alpha}_{i}-\alpha_{i}\right)\left(\hat{\alpha}_{i}-\alpha_{i}\right) x_{j}{ }^{T} x_{i} \\
\text { s.t. } \sum_{j=1}^{m}\left(\hat{\alpha}_{i}-\alpha_{i}\right) \\
0<\hat{\alpha}_{i}, \alpha_{i}<C
\end{gathered}
$$

The KKT condition is used to solve the duality problem, and the solution of SVR is obtained:

$$
f(x)=\sum\left(\hat{\alpha}_{i}-\alpha_{i}\right) x_{i}^{T} x+b
$$

In this way, the support vector machine regression model is obtained.

\subsection{Clustering and Grading of Wine Grapes}

Is the purpose of clustering task by learning from samples of unmarked training data to reveal the internal nature and law of ontology for wine grapes classification, based on the grapes, and wine after the dimension reduction of physical and chemical index variable clustering for wine grapes classification results, and by calculating the comprehensive scoring average within the cluster of grapes to define levels of baseline score. We tried four different clustering algorithms, including Kmeans clustering, Gaussian mixture clustering, density clustering and hierarchical clustering, to estimate samples, and selected the one with better clustering results by calculating inter-cluster variance. [4]

\subsubsection{K-means Clustering}

Given the sample set $D=\left\{x_{1}, x_{2}, \cdots, x_{p}\right\}$, and the algorithm divides the clusters into $C=\left\{c_{1}, c_{2}, \cdots, c_{p}\right\}$, the minimum squared error is:

$$
\begin{aligned}
& E=\sum_{i=1}^{k} \sum_{x \in C}\left\|x-\mu_{i}\right\|_{2}^{2} \\
& \mu_{i}=\frac{1}{|C|} \sum_{x \in C} x
\end{aligned}
$$

Intuitively, the above equation describes the closeness of the samples in the cluster around the mean vector of the cluster. The smaller the E value is, the higher the similarity of the samples in the cluster is.Therefore, the K-means algorithm is obtained by minimizing the above equation. 


\subsubsection{Gaussian Mixture Clustering}

Different from K-means and LVQ, which use prototype vector to describe the cluster structure, Mixture-of-Gaussian clustering uses probability model to express the cluster model.

According to the definition of the Gaussian distribution, we can define the Gaussian mixture distribution:

$$
p_{M}(x)=\sum_{i=1}^{k} \alpha_{i} \cdot p\left(x \mid \mu_{i}, \tau_{i}\right)
$$

Whereinto, $\mu$ is an n-dimensional mean vector, $\tau$ is an $n \times n$ covariance matrix, and $p(x \mid \mu, \tau)$ is a probability density function. The distribution is composed of $K$ mixed components, each of which corresponds to a Gaussian distribution.

Suppose that the sample generation process is given by the Gaussian mixture distribution: firstly, Gaussian mixture components are selected according to the prior distribution defined by $\alpha$, and then the corresponding samples are generated by sampling according to the probability density of the selected mixture components.Bayes' theorem is used to calculate the prior probability, and the cluster division is determined by the posterior probability of the prototype.

Model parameters are estimated by maximum likelihood:

$$
\operatorname{Max} L L(D)=\operatorname{In}\left(\sum_{j=1}^{m} P_{M}\left(x_{j}\right)\right)=\sum_{j=1}^{m} \operatorname{In}\left(\sum_{j=1}^{m} \alpha_{i} \cdot p\left(x \mid \mu_{i}, \tau_{i}\right)\right)
$$

EM algorithm is used for iterative optimization solution: in each iteration, the posterior probability of each sample belonging to each Gaussian component is calculated according to the current parameters, and then the model parameters are updated according to the Lagrange solution.

\subsubsection{Density Clustering}

Density - Based Clustering (Density - Based Clustering) assumes that the clustering structure can be determined by the density of sample distribution. The algorithm investigates the connectivity of samples from the perspective of sample density, and continuously expands the clustering cluster based on the connected samples to obtain the final clustering results.

DBSCAN is a famous density clustering algorithm, which is based on a set of Neigh-Borhood parameters $(\varepsilon$, MinPts $)$ to describe the tightness of sample distribution.

The DBSCAN algorithm first selects a core object in the data set as a "seed", and then determines the corresponding clustering cluster from this point of view.

The algorithm first finds out all the core objects according to the given neighborhood parameters $(\varepsilon$, MinPts $)$, and then takes any core object as the starting point to find out the sample whose density can be reached to generate the cluster, until all the core objects have been visited.

\subsubsection{Hierarchical Clustering}

Hierarchical Clustering is an attempt to partition a dataset at different levels to form a tree-like cluster structure. The partitioning of data sets can be done with either a "bottom-up" aggregation strategy or a "top down" unbundling strategy.

AGNES algorithm is a hierarchical clustering algorithm that adopts bottom-up aggregation strategy. It first regards each sample in the data set as an initial clustering cluster, and then finds out the two closest clustering clusters in each step of algorithm operation to merge. This process is repeated continuously until the preset number of clustering clusters is reached.

Therefore, the key lies in how to calculate the distance between cluster clusters. In fact, each cluster is a sample set, and it only needs to adopt some distance about the set.

Average distance is used in this paper: 
Volume 15 (2021)

$$
d_{\text {avg }}\left(C_{i}, C_{j}\right)=\frac{1}{\left|C_{i}\right|\left|C_{j}\right|} \sum_{x \in C_{i}} \sum_{z \in C_{j}} \operatorname{dist}(x, z)
$$

At this time, Agnes algorithm based on average distance was also called "average-linkage" algorithm.

\section{Interpretation of Result}

All algorithms in this paper are implemented using Python software.

The calculation results and analysis of the data are as follows:

\subsection{Dimension Reduction Results of Physical and Chemical Indexes of White Grape}

Table 4. Characteristic values and contribution of principal components of white grape

\begin{tabular}{|c|c|c|c|}
\hline Principal component & Eigenvalue & Contribution Degree & Cumulative contribution \\
\hline 1 & 5.387 & 0.199 & 0.199 \\
\hline 2 & 4.812 & 0.178 & 0.377 \\
\hline 3 & 2.777 & 0.102 & 0.480 \\
\hline 4 & 2.117 & 0.078 & 0.559 \\
\hline 5 & 1.83 & 0.067 & 0.627 \\
\hline 6 & 1.568 & 0.058 & 0.685 \\
\hline 7 & 1.448 & 0.053 & 0.738 \\
\hline 8 & 1.264 & 0.046 & 0.785 \\
\hline 9 & 1.0791 & 0.039 & 0.825 \\
\hline
\end{tabular}

Therefore, the first nine Principal components were selected by dimension-reduction, and the cumulative variance contribution at this time exceeded $80 \%$.

\subsection{Comprehensive Score Results of White Grapes}

Table 5. Comprehensive score of physical and chemical indexes of white grapes (standardized)

\begin{tabular}{|c|c|c|c|c|c|c|c|}
\hline sample & 1 & 2 & 3 & 4 & 5 & 6 & 7 \\
\hline score & -1.135 & -0.057 & 1.018 & -0.286 & 0.491 & 0.086 & -0.103 \\
\hline sample & 8 & 9 & 10 & 11 & 12 & 13 & 14 \\
\hline score & -0.934 & 0.436 & 0.167 & -0.190 & 0.171 & -0.757 & -0.571 \\
\hline sample & 15 & 16 & 17 & 18 & 19 & 20 & 21 \\
\hline score & -0.030 & -1.130 & -0.719 & -0.249 & -0.015 & 0.128 & -0.604 \\
\hline sample & 22 & 23 & 24 & 25 & 26 & 27 & 28 \\
\hline score & -0.007 & 0.071 & 1.109 & 0.340 & 0.460 & 1.644 & 0.667 \\
\hline
\end{tabular}

\subsection{Principal Component Analysis of Physical and Chemical Indexes of White Wine}

Table 6. Characteristic values of principal components in white wine

\begin{tabular}{|c|c|c|c|}
\hline Principal Component & Eigenvalue & Contribution degree & Cumulative Contribution \\
\hline 1 & 2.994 & 0.374 & 0.374 \\
\hline 2 & 2.367 & 0.296 & 0.670 \\
\hline 3 & 1.075 & 0.134 & 0.805 \\
\hline 4 & 0.780 & 0.097 & 0.902 \\
\hline
\end{tabular}

Therefore, the first four principal components were selected through dimension-reduction, and the cumulative variance contribution at this time exceeded $90 \%$. 
Table 7. White grape eigenvector matrix

\begin{tabular}{|c|c|c|c|c|}
\hline Physicochemical Indexes & $\begin{array}{c}\text { Principal } \\
\text { Component1 }\end{array}$ & $\begin{array}{c}\text { Principal } \\
\text { Component2 }\end{array}$ & $\begin{array}{c}\text { Principal } \\
\text { Component3 }\end{array}$ & $\begin{array}{c}\text { Principal } \\
\text { Component4 }\end{array}$ \\
\hline Tannin(mmol/L) & -0.526 & -0.06 & 0.089 & -0.221 \\
\hline total phenols $(\mathrm{mmol} / \mathrm{L})$ & -0.543 & -0.067 & 0.099 & -0.001 \\
\hline Wine flavonoids $(\mathrm{mmol} / \mathrm{L})$ & -0.379 & 0.131 & 0.115 & 0.724 \\
\hline resveratrol(mg/L) & 0.149 & -0.032 & 0.887 & 0.194 \\
\hline $\begin{array}{c}\text { DPPH half inhibited volume } \\
\text { IV50) } 1 / \mathrm{IV50}(\mathrm{uL})\end{array}$ & -0.471 & -0.016 & 0.14 & -0.447 \\
\hline colourL*(D65) & 0.082 & 0.569 & 0.263 & -0.301 \\
\hline coloura*(D65) & -0.182 & 0.49 & -0.303 & 0.29 \\
\hline colourb*(D65) & -0.034 & -0.64 & -0.042 & 0.125 \\
\hline
\end{tabular}

\subsection{Regression Results of Subjective Score of White Wine}

\subsubsection{Regression Results of Decision Tree}

Sample data were randomly divided into training set 4 and test set 1 , and the regression decision tree was obtained as shown in the figure 1:

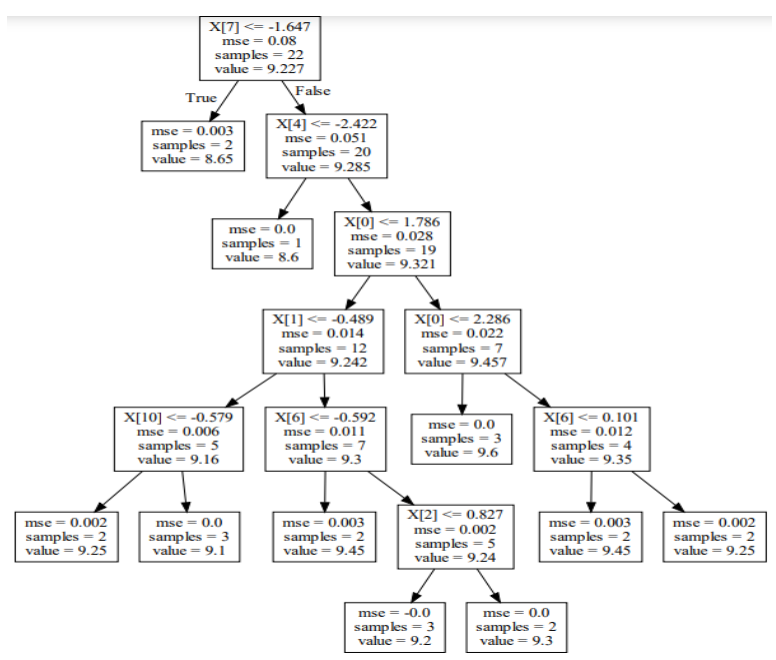

Figure 1. White Grape Regression Decision Tree

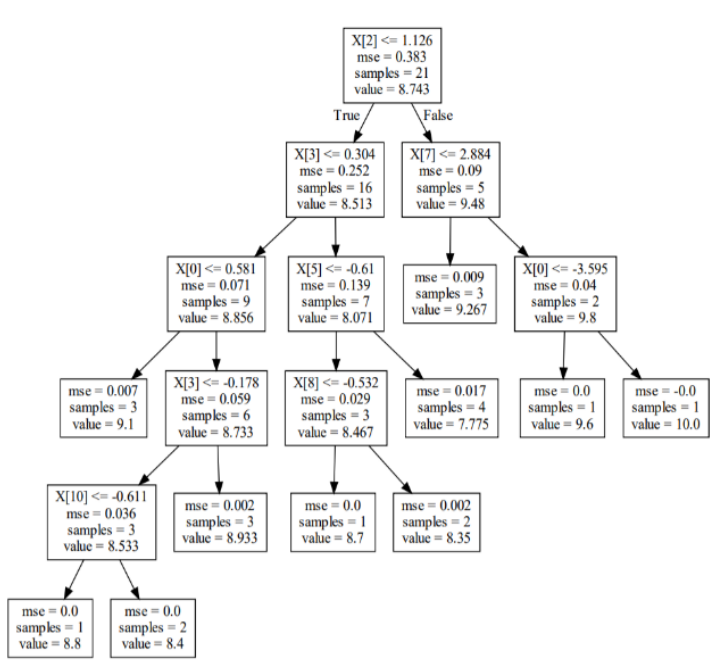

Figure 2. Red grape regression decision tree

The mean square error of the decision tree model on the training set is 0.0014 , while the mean square error on the test set is 0.112 . Therefore, the regression result of this model is good, and we choose this model.

\subsubsection{Support Vector Regression Results}

Sample data were randomly divided into training set 4 and test set 1 , and the meeting scale of support vector was obtained.

The mean square error of this model on the training set is 0.0102 , while the mean square error on the test set is 0.148 . The regression result of this model is worse than that of the decision tree model, so we reject the result of this model.

\subsection{Clustering and Grading Results of White Wine}

According to the traditional rules of the wine industry, we clustered the grapes into four different grades and graded them according to the overall score of the grapes in the clusters. 
Because it is a high-dimensional clustering, the text only outputs the result graph of hierarchical clustering, and other methods are not suitable for the graph.

\subsubsection{K-means Clustering Results}

Table 8. K-means clustering results

\begin{tabular}{|c|c|c|c|c|c|c|c|}
\hline Sample Number & 19 & 17 & 28 & 2 & 3 & 9 & 4 \\
\hline labels & 0 & 0 & 0 & 0 & 0 & 0 & 0 \\
\hline Sample Number & 14 & 26 & 25 & 23 & 22 & 21 & 20 \\
\hline labels & 0 & 0 & 0 & 0 & 0 & 0 & 0 \\
\hline Sample Number & 5 & 12 & 27 & 15 & 24 & 10 & 11 \\
\hline labels & 0 & 1 & 1 & 1 & 1 & 1 & 2 \\
\hline Sample Number & 8 & 16 & 13 & 1 & 7 & 18 & 6 \\
\hline labels & 2 & 2 & 2 & 2 & 3 & 3 & 3 \\
\hline
\end{tabular}

Table 9. K-means benchmark points at all levels

\begin{tabular}{|c|c|c|}
\hline Rank & Count & Score \\
\hline 1 & 2 & 1.376 \\
\hline 2 & 16 & 0.095 \\
\hline 3 & 7 & -0.289 \\
\hline 4 & 3 & -0.752 \\
\hline
\end{tabular}

\subsubsection{Density Clustering Results}

The results based on DBSCAN algorithm group all samples into one group, so this result is discarded.

\subsubsection{Hierarchical Clustering Results}

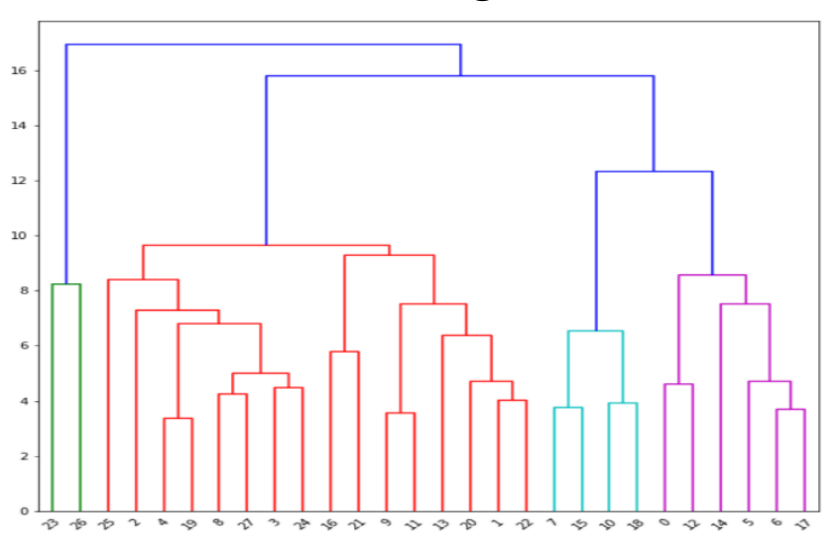

Figure 3. Cluster diagram of white grape Agnes

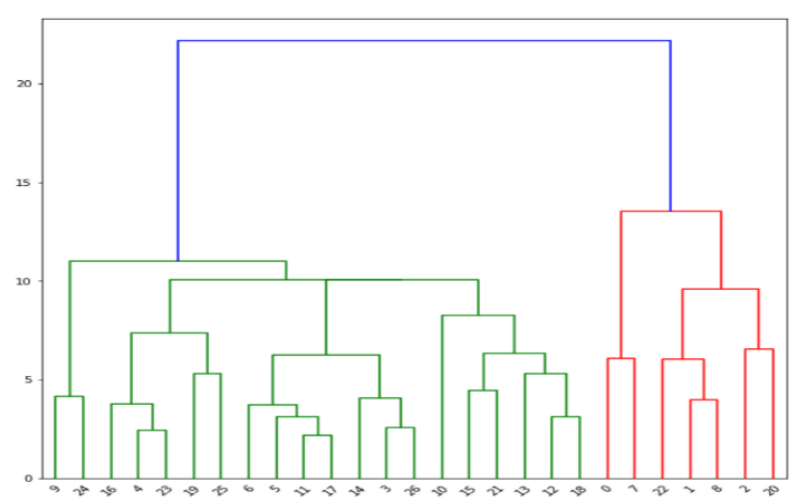

Figure 4. Cluster diagram of red grape Agnes

In the end, we chose the clustering results of Agnes. Therefore, under this model, the variance of benchmark scores among grape grades was the largest, and the sample distribution was reasonable.

\section{Robustness Test}

In order to verify the robustness of all the models in this paper, we carried out the above model and solution on the same data of red grapes.

The results show that our model is robust. The data analysis is as follows: 


\subsection{Dimension Reduction Results of Physical and Chemical Indexes of Red Grape}

Table 10. Eigenvalue and contribution degree of principal components of red grape

\begin{tabular}{|c|c|c|c|}
\hline Principal Component & Eigenvalue & Contribution Degree & Cumulative contribution \\
\hline 1 & 6.644 & 0.246 & 0.246 \\
\hline 2 & 4.332 & 0.160 & 0.407 \\
\hline 3 & 2.954 & 0.109 & 0.516 \\
\hline 4 & 2.898 & 0.107 & 0.623 \\
\hline 5 & 2.000 & 0.074 & 0.697 \\
\hline 6 & 1.603 & 0.059 & 0.757 \\
\hline 7 & 1.233 & 0.046 & 0.802 \\
\hline
\end{tabular}

Through dimension-reduction, the first seven principal components were selected, and the cumulative variance contribution at this time exceeded $80 \%$.

\subsection{Comprehensive Score Results of White Grapes}

Table 11. Comprehensive score of physical and chemical indexes of red grapes (standardized)

\begin{tabular}{|c|c|c|c|c|c|c|c|}
\hline Item & Value & Value & Value & Value & Value & Value & Value \\
\hline Score & -1.009 & -1.042 & -1.658 & 0.394 & 0.522 & 0.337 & -0.036 \\
\hline Sample & 8 & 9 & 10 & 11 & 12 & 13 & 14 \\
\hline Score & -0.994 & -0.823 & 1.327 & -0.557 & 0.104 & 0.011 & -0.608 \\
\hline Sample & 15 & 16 & 17 & 18 & 19 & 20 & 21 \\
\hline Score & 0.528 & -0.386 & 0.547 & 0.346 & 0.140 & 0.727 & -0.995 \\
\hline Sample & 22 & 23 & 24 & 25 & 26 & 27 & 28 \\
\hline Score & -0.431 & 0.265 & 0.453 & 1.517 & 0.897 & 0.423 & 0.000 \\
\hline
\end{tabular}

\subsection{Principal Component Analysis Results of Physical and Chemical Indexes of Red Wine}

Table 12. Eigenvalues of principal components in red grape

\begin{tabular}{|c|c|c|c|}
\hline Principal Component & Eigenvalue & Contribution degree & Cumulative contribution \\
\hline 1 & 4.774 & 0.530 & 0.530 \\
\hline 2 & 1.846 & 0.205 & 0.736 \\
\hline 3 & 0.789 & 0.088 & 0.823 \\
\hline 4 & 0.751 & 0.083 & 0.907 \\
\hline
\end{tabular}

Therefore, the first four principal components were selected through dimension-reduction, and the cumulative variance contribution at this time exceeded $90 \%$.

Table 13. Red wine eigenvector matrix

\begin{tabular}{|c|c|c|c|c|}
\hline Physicochemical Indexes & $\begin{array}{c}\text { Principal } \\
\text { Component }\end{array}$ & $\begin{array}{c}\text { Principal } \\
\text { Component2 }\end{array}$ & $\begin{array}{c}\text { Principal } \\
\text { Component3 }\end{array}$ & $\begin{array}{c}\text { Principal } \\
\text { Component4 }\end{array}$ \\
\hline Tannin (mmol/L) & 0.404 & 0.232 & -0.107 & -0.307 \\
\hline total phenols (mmol/L) & 0.424 & -0.049 & 0.064 & 0.151 \\
\hline Wine flavonoids (mmol/L) & 0.441 & -0.086 & 0.135 & 0.131 \\
\hline resveratrol(mg/L) & 0.258 & 0.365 & -0.484 & -0.111 \\
\hline $\begin{array}{c}\text { DPPH half inhibited } \\
\text { volume(IV50) 1/IV50(uL) }\end{array}$ & 0.190 & -0.484 & 0.433 & -0.021 \\
\hline colourL*(D65) & 0.425 & -0.150 & 0.160 & 0.181 \\
\hline coloura*(D65) & -0.397 & 0.154 & 0.266 & 0.334 \\
\hline colourb*(D65) & -0.149 & -0.519 & -0.157 & -0.674 \\
\hline
\end{tabular}




\subsection{Regression Results of Subjective Score of White Wine}

\subsubsection{Regression Results of Decision Tree}

Sample data were randomly divided into training set 4 and test set 1 , and the regression decision tree was obtained as shown in the figure 2 above. The mean square error of the decision tree model on the training set was 0.0072 , while the mean square error on the test set was 0.5499 . Therefore, the regression result of this model was good, and we chose this model.

\subsubsection{Support Vector Regression Results}

Sample data were randomly divided into training set 4 and test set 1 , and the meeting scale of support vector was obtained. The mean square error of this model on the training set is 0.0376 , while the mean square error on the test set is 0.18085 . The regression result of this model is worse than that of the decision tree model, so we reject the result of this model.

\subsection{Clustering and Grading Results of White Grapes}

According to the traditional rules of the wine industry, we clustered the grapes into four different grades and graded them according to the overall score of the grapes in the clusters.Because it is a high-dimensional clustering, the text only outputs the result graph of hierarchical clustering, and other methods are not suitable for the graph.

\subsubsection{Hierarchical Clustering Results}

In the end, we chose the clustering results of Agnes. Therefore, under this model, the variance of benchmark scores among grape grades was the largest, and the sample distribution was reasonable. Analysis of red wine data proves that our model is robust.

\section{Improvement and Extension of the Model}

In the second question, we directly used the mean value of the overall score of wine given by each taster as the approximate estimate of the quality of wine sample, and used it as the explained variable of regression, which was rough.The model can be further improved to establish a comprehensive wine quality evaluation system. The evaluation system mainly contains two indicators:

The first aspect is the quality of the wine.This includes appearance, aroma, taste, and overall score.Appearance includes clarity and tone, aroma includes purity, concentration and quality, and taste is reflected by purity, concentration, durability and quality.

The second aspect is the physical and chemical indexes of wine grape itself.For example, 27 indexes such as total flavonoids, total phenols, tannin and fruit peel quality in addition 2.For the physical and chemical indexes of the 27 wine grapes themselves, the internal relationship was analyzed according to the data obtained from multiple samples, and the significantly correlated indexes were combined to make the calculation simple.Based on the above analysis, a comprehensive evaluation index system can be constructed, and a model can be established for the comprehensive evaluation of multiple indicators.Based on the result of comprehensive evaluation, wine grapes can be graded.In addition, in the case of index dimensionality reduction, there are multiple physical and chemical indicators in wine grape and wine respectively. If the simple correlation analysis method is adopted, the correlation between single $\mathrm{X}$ and single $\mathrm{Y}$ is only considered in isolation, but the correlation among the variables within the $\mathrm{X}$ and $\mathrm{Y}$ variable sets is not considered.The chemical process by which wine grapes are fermented into wine makes many simple correlation coefficients between the two groups of variables, which makes the problem seem complicated and difficult to describe as a whole.Therefore, the multivariate statistical method--canonical correlation analysis, which is used to study the correlation between the two groups of variables, is considered to identify and quantify the relationship between the two groups of variables of wine grape and the physical and chemical indicators of wine, consider the linear combination of the two groups of variables, and study the correlation coefficient between them. 


\section{Conclusion}

In the study of this paper, with wine rating as the dependent variable and wine physical and chemical indexes as the independent variables, the physical and chemical indexes of wine grapes were filtered by using the method of principal component analysis to reduce the dimensionality, and the functional relationship between wine rating and wine physical and chemical indexes was established by using the method of decision tree regression. Then, in this paper, after comparing the results of four clustering algorithms, the wines were clustered and analyzed based on the hierarchical clustering method of AGNES algorithm. Considering the facts as well as the rationality of grading, both red and white wines were divided into 4 levels, and the estimated scores of the wines at each level were averaged as the benchmark scores of the wines at that level. The mechanism of the influence of physicochemical indicators on wine quality was illustrated. The physicochemical composition of wine grapes indirectly influences the quality of wine by affecting the physicochemical composition of wine.

\section{References}

[1] Liu Bingbing, Song Di. Application of multiple regression analysis for wine quality evaluation modeling [J]. Journal of Anqing Normal College (Natural Science Edition),2014,20(04):29-35.

[2] Zhang J, Ma Xiu Da, Liu X, Liu SY, Yu QING. Research on the evaluation method of red wine based on Principal Component analysis [J]. Journal of Northeastern Electric Power University,2013,33(06):41-44.

[3] Wang Dingcheng, Fang Tingjian, Gao Lifu, Ma Yongjun. Support vector machine regression online modeling and applications [J]. Control and Decision Making,2003(01):89-91+95.

[4] Sun Jigui, Liu Jie, Zhao Lianyu. Research on clustering algorithm[J]. Journal of Software,2008(01):4861. 